MODELING, IDENTIFICATION AND CONTROL, 1983, VOL. 4, NO. 2, 95-100

doi:10.4173/mic.1983.23

\title{
How to drive a delayed response, stochastic system close to equilibrium
}

\author{
SJUR D. FLÅM†
}

Keywords: stability, control, stochastic system, delayed differential equation.

\begin{abstract}
We consider a continuous system that, in the absence of random perturbations and external forcing, is uniformly, asymptotically stable in a global sense. The system evolution is governed by a retarded differential equation. To this equation is added unidentified, time- and state-dependent noise. However, in each instance the noise is bounded over the probability space. We demonstrate that approximate stability is obtainable by a deterministic approach based on worst case analysis.
\end{abstract}

\section{Introduction}

In a recent paper Corless and Leitmann (1981) studied stability control of a stochastic dynamic system as described by an ordinary differential equation. They assumed that the system when subject to neither control nor noise, had a globally, uniformly stable steady state. Both the control and the noise, when present, enter additively in their model. Furthermore, the unknown noise is bounded by a known function of time and state. In this set-up they designed a continuous feedback control guaranteeing that the system will ultimately settle within any prescribed neighbourhood of the equilibrium. The objective of this paper is to demonstrate similar although somewhat weaker results when the system response is retarded. This generalization seems appropriate when considering the increased use of delayed differential equations as witnessed for example in biology (Cushing 1970, 1980). The model is formulated in $\S 2$ and the stability properties of the undisturbed system are listed in $\S 3$. These properties are used to design a class of stabilizing controls as mentioned in $\S 4$. Section 5 then provides statements along with proofs of the resulting approximate stability.

\section{The model}

We consider the following $n$-dimensional system:

$$
\left.\begin{array}{rl}
\dot{x}(t) & =f\left(t, x_{t}\right)+B(t, x(t))[u(t, x(t))+e(t, x(t), \omega)] \\
x_{t_{0}} & =\phi \in C
\end{array}\right\}
$$

where $t \geqslant t_{0}$ is time, $x(t) \in R^{n}$ is the state, $u(t, x(t)) \in R^{m}$ is the control, $e(t, x(t), \omega)$ is the noise and $\omega$ is the generic random outcome in the probability space $\Omega$. In (1)

$$
f:\left[t_{0}, \infty\right) \times C \rightarrow R^{n}
$$

is a known continuous functional. $C$ is the set of continuous functions from $[-r, 0]$

Received 16 February 1983.

† Chr. Michelsen Institute, Department of Science and Technology, Fantoftvegen 38, 5036 Fantoft. Bergen.

M.I.C. 
to $R^{n}$ and $\phi$ is the relevant prehistory of the system at time $t_{0}$. On $C$ we use the supremum norm denoted by \|\| . On $R^{n}$ we use the Euclidean norm | $\mid$. By $x_{t}$ we mean the function from $[-r, 0]$ to $R^{n}$ defined by $x_{t}(\theta)=x(t+\theta)$. It follows that $x_{t} \in C$ whenever $x(\cdot):[t-r, t] \rightarrow R^{n}$ exists and is continuous.

It is evident from our model specification that we assume delay effects to have a maximum time range $r \geqslant 0$ of effectiveness. If, by way of example,

$$
f\left(t, x_{t}\right)=\int_{-\infty}^{t} g(\tau, x(\tau)) k(t, d \tau)
$$

then we require that the delay term $k(t, \cdot)$ is a measure with support contained in $[t-r, t]$. Note that the system as modelled by (1) responds immediately to changes in control and noise. The after-effects work exclusively through the operator $f$.

Also in (1) $B:\left[t_{0}, \infty\right) \times R^{n} \rightarrow R^{n \times m}$ is a known continuous matrix valued function.

By contrast, the random term $e:\left[t_{0}, \infty\right) \times R^{n} \times \Omega \rightarrow R^{m}$ is unknown. However, and this is important, we assume that $\mid e(t, x(t), \omega \mid \leqslant P(t, x(t))$ almost surely.

Here

$$
P:\left[t_{0}, \infty\right) \times R^{n} \rightarrow(0, \infty)
$$

is some specified function. We suppose throughout that $f, B, e$ and $P$ meet all necessary conditions in terms of boundedness and smoothness in order for the solution of (1) to exist on $\left[t_{0}, \infty\right)$ whenever $u(t, x(t))$ is continuous in $(t, x(t))$ and bounded by $P(t, x(t))$. For specified $u(t, x(t))$ we will denote the solution of (1) by $x\left(t_{0}, \phi\right)(t)$ or simply by $x(t)$ when no confusion arises.

\section{Stability of the undisturbed system}

Consider the system

$$
\dot{x}(t)=f\left(t, x_{t}\right)
$$

where control and noise are absent. $f$ is supposed to possess enough smoothness to ensure that the solution $x\left(t_{0}, \phi\right)(t)$ of (2) satisfying $x_{t_{0}}\left(t_{0}, \phi\right)=\phi$ exists for all $t \geqslant t_{0}$, and depends continuously on $\left(t_{0}, \phi, t\right)$.

We shall assume that (2) is globally asymptotically stable, and without loss of generality, let $x \equiv 0$ be the equilibrium, i.e. $f(t, 0) \equiv 0$. Specifically, we suppose that there exists a Lyapunov function $V: R \times R^{n} \rightarrow R$ and continuous, strictly increasing functions $\gamma_{i}: R_{+} \rightarrow R_{+}$with $\gamma_{i}(0)=0$ for $i=1,2,3$ and $\gamma_{1}(\infty)=\infty$ such that for all $t \in R$, $x \in R^{n}, \phi \in C$ we have

$$
\gamma_{1}\left(|x| \leqslant V(t, x) \leqslant \gamma_{2}(|x|) \text { and } \dot{V}(t, \phi(0)) \leqslant-\gamma_{3}(|\phi(0)|) .\right.
$$

Here

$$
\dot{V}(t, \phi(0)) \stackrel{\text { def }}{=} \lim _{h \rightarrow 0^{+}} \sup \frac{1}{h}[V(t+h, x(t, \phi)(t+h))-V(t, \phi(0))]
$$

Then supposing that $f$ takes $R x$ (bounded sets of $C$ ) into bounded sets of $R^{n}$, we know that $x \equiv 0$ is globally, uniformly asymptotically stable (Hale 1977, Theorem 5.2.1). Hereafter impose the additional assumption that $V(t, x)$ is continuously differentiable. 
It follows that :

$$
\dot{V}(t, \phi(0))=\frac{\partial V(t, \phi(0))}{\partial t}+\frac{\partial V(t, \phi(0))}{\partial x} \cdot f(t, \phi)
$$

\section{The proposed control (Corless and Leitmann 1981)}

Since the undisturbed system (2) is dissipative it is tempting to try to control the stochastic system (1) as guided by the direction of steepest descent in "potential energy' $V(t, x)$. Furthermore, in order to counteract the continual random disturbance, the feedback control should be comparable in norm to $P(x, t)$. Towards this end define for specified $\epsilon>0$ a continuous feedback control $u_{\varepsilon}$ by:

and

$$
\left.u_{\varepsilon}(x, t)=-\frac{\mu(t, x)}{|\mu(t, x)|} P(t, x) \quad \text { if }|\mu(t, x)|>\epsilon\right\}
$$

$$
\left|u_{\varepsilon}(t, x)\right| \leqslant P(t, x) \text { otherwise }
$$

Here $\mu$ is defined by

$$
\mu(t, x)=B^{*}(t, x) \nabla_{x} V(t, x) P(t, x)
$$

It is important to observe that, using this control, along the trajectories of (1) we now have $\dot{V}(t, x) \leqslant-\gamma_{3}(|x|)+2 \epsilon$, by (3).

\section{Stability control}

We first translate the results of Corless and Leitmann (1981) to a special case of delayed equations. Specifically, we make the following

\section{Assumption $I$}

If $|x(t)| \geqslant r$ for all $t \in(a, b)$ where $a<b$, then

$$
\frac{\partial V(t, x(t))}{\partial t}+\frac{\partial V(t, x(t))}{\partial x} \cdot f\left(t, x_{t}\right) \leqslant-\gamma_{3}(r)
$$

Later on this assumption will be dropped. However, when it is valid we have the following statements:

\section{Theorem 1}

Under Assumption I the model (1) with the feedback control (4) has the following property of uniform boundedness. For all $\delta, \epsilon>0$ if $\epsilon<0 \cdot 5 \gamma_{3}(\infty)$ and $\|\phi\| \leqslant \delta$ then $\left|x\left(t_{0}, \phi\right)(t)\right| \leqslant \gamma_{1}^{-1} \gamma_{2}\left(\delta_{\varepsilon}\right)$ for all $t \geqslant t_{0}-r$. Here $\delta_{\varepsilon}=\max \left(\delta, \gamma_{3}^{-1}(2 \epsilon)\right)$.

\section{Theorem 2}

Under Assumption I a solution of (1) applying the control (4) has the following property of uniform ultimate boundedness: For all $\delta, \epsilon>0$ if $\|\phi\| \leqslant \delta$ and $d>$ $\gamma_{1}^{-1} \gamma_{2} \gamma_{3}^{-1}(2 \epsilon)$ then $\left|x\left(t_{0}, \phi\right)(t)\right| \leqslant d$ for all $t \geqslant t_{0}+T(\bar{d}, \delta)$ where

and $\bar{R}=\gamma_{2}^{-1} \gamma_{1}(\bar{d})$.

$$
T(\bar{d}, \delta)=\left\{\begin{array}{cc}
0 \quad \text { if } \delta \leqslant \bar{R} \\
\frac{\gamma_{2}(\delta)-\gamma_{1}(\bar{R})}{\gamma_{3}(\bar{R})-2 \epsilon} & \text { otherwise }
\end{array}\right.
$$


The proof of Theorems 1 and 2 follows verbatim that given by Corless and Leitmann for the case of no delays and will not be reproduced here.

We stress that Assumption I is highly restrictive for systems with proper delays. This provides motivation for us to prove approximate stability under conditions which fit better to retarded equations.

For this purpose we will impose the following alternative assumptions:

\section{Assumption II}

There exists a continuous non-decreasing function $p: R_{+} \rightarrow R_{+}$such that $p(s) \geqslant s$ and

$$
\frac{\partial V(t, \phi(0))}{\partial t}+\frac{\partial V(t, \phi(0))}{\partial x} \cdot f(t, \phi) \leqslant-\gamma_{3}(|\phi(0)|) \text { if } \phi \in C
$$

and $V(t+\theta, \phi(\theta)) \leqslant p(V(t, \phi(0)))$ for all $\theta \in[-r, 0]$.

\section{Assumption III}

Let $p: R_{+} \rightarrow R_{+}$satisfy $p(s) \geqslant s$. There exists a continuous strictly increasing $\gamma_{4}: R_{+} \rightarrow R_{+}$with $\gamma_{4}(0)=0$ such that:

$$
\frac{\partial V(t-r, \phi(0))}{\partial t}+\frac{\partial V(t-r, \phi(0))}{\partial x} f\left(t-r, \phi_{0}\right) \geqslant \gamma_{4}(|\phi(0)|) \text { if } \phi:[-r, r] \rightarrow R^{n}
$$

is continuous and $t \geqslant t_{0}+r$ and $V(t+\theta, \phi(\theta)) \leqslant p(V(t-r, \phi(0))$ for all $\theta \in[0, r]$.

\section{Theorem 3}

Under Assumptions II and III any solution $x\left(t_{0}, \phi\right)(t)$ of (1) with control (4) and $\|\phi\| \leqslant \delta,\left\|x_{t_{0}+r}\left(t_{0}, \phi\right)\right\| \leqslant \delta$ will satisfy $\left|x\left(t_{0}, \phi\right)(t)\right| \leqslant \gamma_{1}{ }^{-1} \gamma_{2}\left(\delta_{\varepsilon}\right)$ for all $t \geqslant t_{0}-r$. Here $\delta_{\varepsilon}=\max \left(\delta, \gamma_{3}^{-1}(2 \epsilon), \gamma_{4}^{-1}(2 \epsilon)\right)$.

\section{Proof}

Define $\bar{V}: R \times C \rightarrow R$ by $\bar{V}(t, \phi)=\sup _{-r \leqslant \theta \leqslant 0} V(t+\theta, \phi(\theta))$.

Note that $\gamma_{1}(|\phi(0)|) \leqslant \bar{V}(t, \phi) \leqslant \gamma_{2}(\|\phi\|)$. Hereafter simply write $x(t)$ for $x\left(t_{0}\right.$, $\phi)(t)$ and $x_{t}$ for $x_{t}\left(t_{0}, \phi\right)$. Now suppose $|x(T)|>\gamma_{1}{ }^{-1} \gamma_{2}\left(\delta_{\varepsilon}\right)$ for some $T>t_{0}+r$. Let

$$
\tau=\inf \left\{s: t_{0}+r \leqslant s \leqslant T \text { and } \dot{\bar{V}}\left(t, x_{t}\right) \leqslant 0 \text { for all } t \in(s, T]\right\}
$$

Here

$$
\dot{\bar{V}}(t, \phi) \stackrel{\text { def }}{=} \lim _{h \rightarrow 0_{+}} \sup \frac{1}{h}\left[\bar{V}\left(t+h, x_{t+h}(t, \phi)\right)-\bar{V}(t, \phi)\right]
$$

First we discuss the case $\tau=t_{0}+r$.

Then $\gamma_{1}(|x(T)|) \leqslant \bar{V}\left(T, x_{T}\right) \leqslant \bar{V}\left(\tau, x_{\tau}\right) \leqslant \gamma_{2}\left(\left\|x_{\tau}\right\|\right) \leqslant \gamma_{2}(\delta) \leqslant \gamma_{2}\left(\delta_{\varepsilon}\right)$.

Thus $|x(T)| \leqslant \gamma_{1}^{-1} \gamma_{2}\left(\delta_{\varepsilon}\right)$, a contradiction.

Hence suppose hereafter that $\tau>t_{0}+r$. For notational convenience write $x_{\tau}\left(t_{0}, \phi\right)=\Phi$. There exists a $\theta_{0} \in[-r, 0]$ such that

$$
\bar{V}(\tau, \Phi)=V\left(\tau+\theta_{0}, \Phi\left(\theta_{0}\right)\right)>V(\tau+\theta, \Phi(\theta)) \text { for all } \theta \in\left(\theta_{0}, 0\right]
$$


We first exclude the possibility that $V(\tau-r, x(\tau-r))$ and $V(\tau, x(\tau))<\bar{V}\left(\tau, x_{\tau}\right)$. In fact, this condition would imply that

$$
\bar{V}\left(\tau-h, x_{\tau-h}\right)=\bar{V}\left(\tau, x_{\tau}\right)=\bar{V}\left(\tau+h, x_{\tau+h}\right), \text { for all } h \in[0, \bar{h}]
$$

where $h>0$ is sufficiently small. Thus $\dot{\bar{V}}\left(\tau-h, X_{\tau-h}\right)=0$ for all $h \in[0, h]$ contradicting the minimality of $\tau$.

Next we discuss the case $V(\tau-r, x(\tau-r))=\bar{V}\left(\tau, x_{\tau}\right)$.

Suppose in addition that $|x(\tau-r)|>\gamma_{4}^{-1}(2 \epsilon)$.

Then by Assumption III we have $\dot{V}(\tau-r, x(\tau-r))>0$.

This contradicts the maximality of $V(\tau-r, x(\tau-r))$.

If on the other hand $|x(\tau-r)| \leqslant \gamma_{4}{ }^{-1}(\epsilon)$ we have

$$
\gamma_{1}(|x(T)|) \leqslant \bar{V}\left(T, x_{T}\right) \leqslant \bar{V}\left(\tau, x_{\mathrm{r}}\right)=V(\tau-r, x(\tau-r)) \leqslant \gamma_{2}(|x(\tau-r)|) \leqslant \gamma_{2}\left(\delta_{\varepsilon}\right)
$$

This gives the contradiction

$$
|x(T)| \leqslant \gamma_{1}^{-1} \gamma_{2}\left(\delta_{3}\right)
$$

The remaining case when

$$
V(\tau-r, x(\tau-r))<\bar{V}\left(\tau, x_{\tau}\right)=V(\tau, x(\tau))
$$

is treated similarly. This completes the proof.

\section{Theorem} $s>0$.

Consider the system (1) satisfying Assumptions II and III with $p(s)>s$ when

For any specified $\Delta>0$ let $\mu=\gamma_{2}{ }^{-1} \gamma_{1}(\Delta)$ and choose a positive $\epsilon<0 \cdot 5 \gamma_{3}(\mu)$, $0 \cdot 5 \gamma_{4}(\mu)$. Then for any initial condition $\phi$ with $\|\phi\| \leqslant \delta>0$ by using the control (4) the solution of (1) will satisfy $|x(t)| \leqslant \Delta$ for all $t \geqslant t_{0}$ if $\delta \leqslant \mu$, and $|x(t)| \leqslant \Delta$ for some $t \leqslant t_{0}+T$ if $\delta>\mu$. Here

$$
T=\frac{N \gamma_{2} \gamma_{1}^{-1} \gamma_{2}\left(\delta_{\varepsilon}\right)}{\gamma_{3}(\mu)-2 \epsilon}
$$

where $N$ is determined as follows. Find $a>0$ such that $p(s)>s+a$ for all $\gamma_{1}(\mu) \leqslant s \leqslant$ $\gamma_{1} \gamma_{2}^{-1} \gamma_{2}\left(\delta_{\varepsilon}\right)$ and let $N$ be the smallest integer such that $\gamma_{1}(\mu)+N a \geqslant \gamma_{2} \gamma_{1}^{-1} \gamma_{2}\left(\delta_{\varepsilon}\right)$.

\section{Proof}

If $\delta \leqslant \mu$ we have $|x(t)| \leqslant \gamma_{1}^{-1} \gamma_{2}\left(\delta_{\varepsilon}\right) \leqslant \gamma_{1}^{-1} \gamma_{2}(\mu)=\Delta$ for all $t \geqslant t_{0}$ by Theorem 3 . Now suppose $\delta>\mu$. Note that $\Delta \geqslant \mu$.

The purpose is to show that $|x(t)| \leqslant \Delta$ for at least one $t \in\left[t_{0}, t_{0}+T\right]$.

This will be true if we demonstrate that $V(t, x(t)) \leqslant \gamma_{1}(\mu)$ for some $t \in\left[t_{0}, t_{0}+T\right]$. By Theorem 3

$$
V(t, x(t)) \leqslant \gamma_{2}\left(\mid x(t \mid) \leqslant \gamma_{2} \gamma_{1}^{-1} \gamma_{2}\left(\delta_{\varepsilon}\right) \text { for all } t \geqslant t_{0}-r\right.
$$

Define the function $\nu: R_{+} \rightarrow R_{+}$by $\nu=\gamma_{2} \gamma_{1}^{-1} \gamma_{2}$. Choose $a>0$ such that $p(s)-s>a$ whenever $\gamma_{1}(\mu) \leqslant s \leqslant \nu\left(\delta_{\varepsilon}\right)$. Let $N$ be the first non-negative integer such that $\gamma_{1}(\mu)+$ $N a>\nu\left(\delta_{\varepsilon}\right)$.

Define $\gamma=\gamma_{3}(\mu)-2 \epsilon$ and $T=N \nu\left(\delta_{\varepsilon}\right) / \gamma$.

Suppose $|x(t)| \geqslant \mu$ and $V(t, x(t))>\gamma_{1}(\mu)+(N-1) a$ on some interval $\left[t_{0}, t_{0}+\tau\right)$. Then $t \in\left[t_{0}, t_{0}+\tau\right)$ implies

$$
p\left(V(t, x(t))>V(t, x(t))+a>\gamma_{1}(\mu)+N a \geqslant \nu\left(\delta_{\varepsilon}\right) \geqslant V(t+\theta, x(t+\theta))\right.
$$


By Assumption II $\dot{V}(t, x(t)) \leqslant-\gamma_{3}(|x(t)|)+2 \epsilon \leqslant-\gamma_{3}(\mu)+2 \epsilon=-\gamma$. Consequently, $V(t, x(t)) \leqslant V\left(t_{0}, x\left(t_{0}\right)\right)-\gamma\left(t-t_{0}\right) \leqslant \nu\left(\delta_{\varepsilon}\right)-\gamma\left(t-t_{0}\right)$.

The positivity of $V$ implies that $\nu\left(\delta_{\varepsilon}\right)-\gamma\left(t-t_{0}\right) \geqslant 0$ and therefore $\tau \leqslant \nu\left(\delta_{\varepsilon}\right) / \gamma$. It follows that $V(t, x(t)) \leqslant \gamma_{1}(\mu)+(N-1) a$ for $t \geqslant t_{0}+v\left(\delta_{\varepsilon}\right) / \gamma$ as long as $|x(t)| \geqslant \mu$.

Inductively assume that $\gamma_{1}(\mu)+(N-k-1) a \leqslant V(t, x(t)) \leqslant \gamma_{1}(\mu)+(N-k) a$ for all $t \in\left[t_{k}, t_{k}+\tau\right)$ where $t_{k} \geqslant t_{0}+k v\left(\delta_{\varepsilon}\right) / \gamma$.

Then if $|x(t)| \geqslant \mu$ in this interval we prove as above that $\tau \leqslant v\left(\delta_{\varepsilon}\right) / \gamma$. Also $V(t, x(t))) \leqslant \gamma_{1}(\mu)+(N-(k+1) a)$ for $t \leqslant t_{k}+\nu\left(\delta_{\varepsilon}\right) / \gamma$ provided $|x(s)| \geqslant \mu$ for all $s \in\left[t_{k}+\nu\left(\delta_{\varepsilon}\right) / \gamma, t\right]$.

It follows that $|x(t)| \leqslant \mu$ for some $t \leqslant t_{0}+T$ or $V\left(t_{0}+T, x\left(t_{0}+T\right)\right) \leqslant \gamma_{1}(\mu)$. Q.E.D.

\section{Concluding remarks}

We have shown that knowledge of a Lyapunov function may furnish a method of stability control of a stochastic system with bounded noise and retarded response. It should be stressed that the proposed control depends only on the bounds of the random noise and not upon its distribution. The actual cost of implementing the control depends very much on the gradient of the Lyapunov function. We have confined ourselves to situations where a Razumikhin type of stability applies (Hale 1977). The more general case requiring functionals and not only functions, seems more difficult. For related studies on control of uncertain systems see Leitmann (1981), Barmish (1982) and Gutman (1979).

\section{REFERENCES}

BARMISH, B. R. (1982). Global and point controllability of uncertain dynamical systems. IEEE Trans. on Automatic Control, 27, 399-407.

Corless, M. J., and LeitmanN, G. (1981). Continuous state feedback guaranteeing uniform, ultimate boundedness for uncertain dynamic systems. IEEE Trans. on Automatic Control, 26, 1139-1144.

Cushing, J. M. (1980). Stability and maturation periods in age-structured populations. In Differential Equations and Applications in Ecology, Epidemics and Population Problems, Ed. Busenberg and Cooke (New York: Academic Press).

Gutman, S. (1979). Uncertain dynamical systems-A Lyapunov min-max approach. IEEE Trans. on Automatic Control, 24, 437-443.

HALE, J. (1977). Theory of functional differential equations (New York: Springer-Verlag).

LeitmanN, G. (1981). On the efficacy of non-linear control in uncertain linear systems. Journal of Dynamical Systems, Measurement and Control, 102, 95-102. 